\title{
Effect of Implementing Suggested Nursing Teaching Protocol on Patients' Outcomes with Mandibular Fracture
}

\author{
Fatma Gareh Ahmed Megahed , Salah El-Dein Gaber Shaltout , Sahra Zaki Azer \\ Demonstrator, Adult Nursing , Faculty of Nursing, Assiut University, Assiut, Egypt. \\ Professor, of Oral and Maxillofacial Surgery, Faculty of Medicine, Assiut University, Assiut, Egypt. \\ Lecture ,of Medical-Surgical Nursing, Faculty of Nursing, Assiut University, Assiut, Egypt.
}

\begin{abstract}
Mandibular fracture is the most common facial fractures and it is an important surgical condition in the world. Aims of the study; assess patients' knowledge about nutrition, oral care and jaw exercises, design, implement and evaluate the effect of suggested nursing teaching protocol on patients' outcomes with mandibular fracture. Research design; A quasiexperimental research design was utilized on a convenient sample of 60 adult patients divided into two equal groups (study and control) 30 patients for each. Tools; I) Patient with mandibular fracture an interview schedule. II) Patient assessment schedule. Results; There was statistically significant difference between study and control groups post implementing nursing teaching protocol as regard knowledge level of patients about nutrition, oral care, and jaw exercises. There was statistically significant difference between study and control group as regards late complications. Conclusion; Patients' knowledge regarding nutrition, oral hygiene and jaw exercises were inadequate. So a specialized nursing teaching protocol was designed, implemented and evaluated. Study group showed improvement in wound healing, maintain normal body weight and decreased incidence of complications while control group showed increase incidence of these complications. Recommendations; Simple illustration booklets should be available for patients to provide them with simple explanation about safely living.
\end{abstract}

\section{Keywords: Fractures, Mandibular, Nursing, Protocol, Teaching}

\section{Introduction:}

The mandible or lower jaw is a horseshoe shaped bone which articulates with the base of the skull on each end. Mandibular fractures are break through mandibular bone. Mandibular fractures are the commonest fractures encountered in maxillofacial trauma after nasal fractures. the position, prominence, anatomic configuration, mobility and less bone support of the mandible make it one of the most frequent facial bones to be fractured. The most commonly fractured sites are the body, condyle, angle, symphysis, ramus and coronoid. The cause of the injury may be road traffic accidents, assault, falls, animal kick, home accidents, industrial injuries or sports injuries but the relative number of each varies considerably between countries and areas. Mandibular fractures are a common form of facial injury in adults and occur most frequently in males during the third decade of life (Adeyemo et al. 2008).

Mandibular fractures may present with a variety of signs and symptoms such as pain, swelling, bleeding, jaw stiffness, difficulty opening or closing the mouth, lose or damaged teeth, tenderness, step deformity at the fracture site, intraoral bruising, gingival lacerations, sublingual hematoma and diminished or altered sensation to the lower lip that resulting from damage to the inferior alveolar nerve (Adams, 2012).
Complications of mandibular fractures (patients' outcomes) include airway blockage, bleeding, difficulty eating, difficulty talking, infection, temporomandibular joint (TMJ) pain, numbness of part of the jaw or face, problems aligning the teeth, swelling, malocclusion, injury to inferior alveolar nerve or mental nerve, malunion and nonunion (Flint et al., 2014).

Wound infection is the most common complication in all types of mandibular fractures. Other complications that occur less often, include malocclusion, nonunion, malunion, tooth loss, trismus, ankyloses, deviation, unsightly scars, and paresthesia. Normal bony union of mandible takes place over 4 - 8 weeks, depending on the age of the patient. A nonunion occurs when bony union has not occurred within this time period (Ambreen and Shah, 2001).

Treatment is aimed at realignment (reduction) of the fractured segments into their normal anatomic positions and prevention of movement by immobilization (fixation) of the fractured bone thereby allowing osseous union to occur. The ultimate goal is to restore occlusion (bite), mandibular anatomy and jaw function. Fractures of the mandible were diagnosed on the basis of history, clinical examination, and radiographs (anteroposterior, lateral, and orthopantogram views) (Bank and Brown, 2001). 
Nurse play an important role about the post treatment care of closed or open reduction to reduce complications included nutrition consultation of jawwire diet, oral hygiene is stressed including daily brushing of the teeth and arch bars using small toothbrush, stop smoking, gum is used to protect the buccal mucosa from the sharp edges of the wires and arch bars, and physiotherapeutic jaw exercises include opening and closing, lateral movements and gum chewing exercises have been described as successful treatments to avoid complications (patients' outcomes) as temporomandibular joint (TMJ) stiffness and trismus. Patients were assessed weekly until bone healing and arch bar removed to observe any pain at the fracture site, wound infection, paresis in the distribution of mandibular or inferior alveolar nerves, malocclusion, and maintenance of maxillomandibular fixation (MMF). Orthopantogram (OPG) was repeated post operatively to determine evidence of bony healing (Subhashraj et al., 2007). The nursing teaching protocol developed by the researcher consists of three parts; Nutritional teaching protocol to prevent weight loss and promote wound healing includes healthy foods, drinks and the way they're made, information about the oral hygienic care and daily activities to control infection: mouth and lip care and jaw exercises include opening and closing, lateral movements and chewing exercises.

Significance of the study:

Maxillofacial trauma is a major cause of mortality and morbidity worldwide (Chrcanovic et al., 2004). Mandibular fractures are one of the most common facial fractures. About 400 patients admitted in trauma unit at Assiut University Hospital according to hospital record 2014. From the researcher's clinical experience in the surgical unit has been observed that the patients who treated with intermaxillary fixation (IMF) needed special nursing teaching protocol to improve the patients' outcomes. This study will be conducted to provide patients with nursing teaching protocol that provide information on how to stay as healthy as possible.

\section{Aims of this study were}

1. To assess patients' knowledge about nutrition, oral care, and jaw exercises.

2. To design suggested nursing teaching protocol for patients with mandibular fracture.

3. To implement suggested nursing teaching protocol for patients with mandibular fracture.

4. To evaluate the effect of suggested nursing teaching protocol on patients' outcomes with mandibular fracture.

\section{Hypothesis}

To fulfill the aim of the study the following research hypothesis were formulated:-
1. The knowledge of the studied group patients after application of suggested nursing teaching protocol will be higher than the control group.

2. Post-operative complications associated with mandibular fracture will be less among the studied group after application of nursing teaching protocol than the control group.

\section{Subjects and Method: \\ Research design}

Quasi- experimental design was utilized to conduct this study.

\section{Setting}

The study was conducted in the trauma department and maxillofacial surgery outpatient clinic at Assiut University Hospital.

\section{Subjects}

A convenience sample of 60 adult patients of both sexes in the trauma department and maxillofacial surgery outpatient clinic at Assiut University Hospital. Age ranges from 18 to 65 years. Patients assigned randomly into two equal groups (study and control) 30 patients for each. The study group received the suggested nursing teaching protocol while the control group received the routine hospital care.

\section{Tools}

Two tools were used in this study and developed by the researcher to collect the necessary data for this study.

Tool (I): An interview schedule for patient of mandibular fracture: to assess demographic data, medical data, and knowledge regarding nutrition, oral care and jaw exercises developed by the researcher based on literature review.

\section{This tool will consist of four parts:-}

Part (1): Demographic data for patients as (name, age, gender, marital status, level of education and occupation).

Part (2): Patient's medical data: This part includes the following items:

A. Health habits: such as use of tea, coffee, alcohol, smoking, drugs and exercise.

B. Medical history: as diabetes, hypertension, previous mandibular trauma, heart Disease, kidney disease, bone tumor, chronic obstructive pulmonary disease (COPD) and asthma.

\section{C- Present health history:}

- Causes of mandibular fracture (Causes of trauma): included road traffic accidents (motor cycle and motor car accidents), fire arm injury, fall from height, industrial accidents, interpersonal violence, sports accidents, animal kick and home accidents.

- Signs and symptoms of mandibular fracture: included pain, swelling, bleeding, facial bruising, limited jaw movement, malocclusion, 
step deformity, tenderness, diminished or altered sensation to lower lip (numbness) and loose or damaged teeth.

Part (3): knowledge assessment schedule: about (nutrition, oral care, and jaw exercises).

A. Nutrition included: Assess patient knowledge related to nutrition as important, types and preparation of food. Assess patient weight loss after postoperative period of intermaxillary fixation.

B. Oral care included: Assess patient knowledge about oral care as benefits, equipment used, different techniques for brushing teeth and frequency.

C. Jaw exercises included: Assess patient knowledge about jaw exercises as importance of these exercise when must do these exercises?, types and techniques of jaw exercises.

Scoring system: As regard patient assessment knowledge about nutrition, oral care and jaw exercises which included 15 items, each item was observed, categorized and scored into either yes $=1$ or no $=0$ on all items.

- Total score divided into satisfactory $\geq 60 \%$

- Unsatisfactory $<60 \%$

Part (4): Nutritional status assessment: pre (during hospitalization) and post implementing nursing teaching protocol (after 2 weeks from discharge) included height, weight, appetite, route of food intake, level of dependency, diet allergy, presence of nutritional disorders.

Tool (II): Patient with mandibular fracture assessment schedule: An observation for complications was designed based on related literature. the observation was performed to evaluate the effect of implementing suggested nursing teaching protocol on patients 'outcomes with mandibular fracture during hospitalization and follow up for 2 weeks then 4 weeks post discharge to observe late complications and patients 'outcomes such as (accommodation of feeding, difficulty talking, periodontitis, gingivitis, wound infection, temporomandibular joint painful movement, malocclusion, injury to inferior alveolar nerve, injury to mental nerve, malunion, nonunion), height and weight, laboratory investigation included (complete blood count).

Suggested nursing teaching protocol:

The nursing teaching protocol was developed in simple Arabic language by the researcher based on patient's needs assessment, literature review, researcher experience, and opinion of the medical and nursing expertise to evaluate effect of nursing teaching protocol on patients' outcomes and consists of three parts:
- Part (1): Nutritional teaching protocol to prevent weight loss and promote wound healing includes healthy foods, drinks and the way they're made. All foods must be blended in a liquid form, thin enough to go through a syringe or straw. After the first 6 weeks patient can progress slowly to a normal diet. It is important to consume enough calories and protein to prevent weight loss and to promote healing after surgery. patient need to have 3 meals and 3 snacks daily. It is important to eat from a variety of food groups.

- Part (2): Information about the oral hygienic care and daily activities to control infection: mouth and lip care. patient should brush teeth after every meal and before bedtime. A small, soft, child size toothbrush is easier to use while jaws are wired together. Brush teeth by taking finger and pulling out the cheek on the side that plan to brush. Take toothbrush and brush in and out. Rinse mouth at least 6 times a day with the saline or salt water provided. Physical activity should be kept to a minimum for at least 6-8 weeks after surgery. Excessive activity (running, exercising, swimming, heavy lifting, house cleaning, contact sports, going up and down stairs quickly) should be avoided.

- Part (3): Jaw exercises include opening and closing, lateral movements and chewing exercises. No recommended any specific exercises during the first week to ten days after surgery. Jaw exercises can be performed between elastic changes. This would involve opening and close the lower jaw, moving the lower jaw side to side, using tongue depressors and chewing gums also help in jaw opening.

Methods of data collection:

- An official permission was obtained from the head of maxillofacial surgery department to conduct the study.

- The study tools and nursing teaching protocol was designed by the researcher after an extensive review of the relevant literature.

- The content validity was done by 5 expertise opinion in medical and nursing field.

- Patient's agreement for voluntary participation was obtained and the purpose and nature of the study were explained.

- Patients meeting the criteria for possible inclusion (their age range between 18-65 years old and the study included patients with isolated mandibular fracture) was approached by the investigator.

- Data was assured confidentiality.

- Privacy and anonymity of patients were ascertained. 
- A pilot study was conducted on $10 \%$ (6) of patients to evaluate the applicability and clarity of tools. According to this pilot study, the required modifications were made and those patients who were involved in the pilot study weren't included in the study.

\section{Ethical considerations:}

- There was no risk for study subject during application of the research.

- Formal consent was obtained from patients or guidance that were participated in the study, after explaining the nature and purpose of the study.

- Confidentiality of patients was assured.

- Study subject had the right to refuse to participate and or withdraw from the study without any rational any time.

- Study subject privacy was considered during collection of data.

\section{Statistical analysis:}

The data were tested for normality using the Anderson-Darling test and for homogeneity variances prior to further statistical analysis. Categorical variables were described by number and percent $(\mathrm{N}$, $\%$ ), where continuous variables described by mean and standard deviation (Mean, SD). Chi-square test and fisher exact test used to compare between categorical variables where compare between continuous variables by t-test. A two-tailed $\mathrm{p}<0.05$ was considered statistically significant. All analyses were performed with the IBM SPSS 20.0 software. 


\section{Results:}

Table(1):Distribution of demographic characteristics and health habits of the study and control groups $(\mathbf{n}=60)$.

\begin{tabular}{|c|c|c|c|c|c|}
\hline \multirow{2}{*}{ Variable } & \multicolumn{2}{|c|}{ Study $(n=30)$} & \multicolumn{2}{|c|}{ Control $(n=30)$} & \multirow{2}{*}{ P. value } \\
\hline & No. & $\%$ & No. & $\%$ & \\
\hline \multicolumn{5}{|l|}{ Age: } & \multirow{6}{*}{0.724} \\
\hline $18-29$ years & 15 & 50.0 & 14 & 46.7 & \\
\hline $30-39$ years & 9 & 30.0 & 6 & 20.0 & \\
\hline $40-49$ years & 2 & 6.7 & 5 & 16.7 & \\
\hline $50-59$ years & 3 & 10.0 & 4 & 13.3 & \\
\hline $60-65$ years & 1 & 3.3 & 1 & 3.3 & \\
\hline Mean \pm SD & \multicolumn{2}{|c|}{$31.47 \pm 12.62$} & \multicolumn{2}{|c|}{$32.6+13.55$} & 0.739 \\
\hline \multicolumn{6}{|l|}{ Gender: } \\
\hline Male & 24 & 80.0 & 23 & 76.7 & \multirow{2}{*}{0.754} \\
\hline Female & 6 & 20.0 & 7 & 23.3 & \\
\hline \multicolumn{6}{|l|}{ Marital status: } \\
\hline Single & 16 & 53.3 & 13 & 43.3 & \multirow{3}{*}{0.545} \\
\hline Married & 12 & 40.0 & 16 & 53.3 & \\
\hline Widowed & 2 & 6.7 & 1 & 3.3 & \\
\hline \multicolumn{6}{|l|}{ Education level: } \\
\hline Illiterate & 8 & 26.7 & 9 & 30.0 & \multirow{6}{*}{0.892} \\
\hline Read and write & 2 & 6.7 & 2 & 6.7 & \\
\hline Primary school & 0 & 0.0 & 1 & 3.3 & \\
\hline Preparatory school & 3 & 10.0 & 4 & 13.3 & \\
\hline Secondary school & 11 & 36.7 & 8 & 26.7 & \\
\hline University & 6 & 20.0 & 6 & 20.0 & \\
\hline \multicolumn{6}{|l|}{ Occupation: } \\
\hline Un employee & 4 & 13.3 & 3 & 10.0 & \multirow{6}{*}{0.854} \\
\hline Drivers & 6 & 20.0 & 9 & 30.0 & \\
\hline Farmer & 7 & 23.3 & 5 & 16.7 & \\
\hline Student & 6 & 20.0 & 7 & 23.3 & \\
\hline House wife & 3 & 10.0 & 4 & 13.3 & \\
\hline Employer & 4 & 13.3 & 2 & 6.7 & \\
\hline \multicolumn{6}{|l|}{ Health habits: } \\
\hline Use of tea/coffee & 26 & 86.7 & 22 & 73.3 & 0.196 \\
\hline Smoking & 15 & 50.0 & 16 & 53.3 & 0.796 \\
\hline Use of alcohol & 0 & 0.0 & 0 & 0.0 & - \\
\hline Exercise & 1 & 3.3 & 0 & 0.0 & 0.313 \\
\hline Drugs(Narcotics) & 0 & 0.0 & 0 & 0.0 & - \\
\hline
\end{tabular}

Table(2): Distribution of patients according to causes of mandibular fracture (cause of trauma) for the study and control groups $(n=60)$.

\begin{tabular}{|c|c|c|c|c|c|}
\hline \multirow{2}{*}{ Causes of mandibular fracture } & \multicolumn{2}{|c|}{ Study $(n=30)$} & \multicolumn{2}{|c|}{ Control $(n=30)$} & \multirow{2}{*}{ P. value } \\
\hline & No. & $\%$ & No. & $\%$ & \\
\hline Road traffic accidents & 15 & 50.0 & 18 & 60.0 & \\
\hline - Motor cycle accidents & 8 & 23.3 & 12 & 36.7 & 0.672 \\
\hline - Motor car accidents & 7 & 16.7 & 6 & 20.0 & \\
\hline Fall from height & 10 & 33.3 & 6 & 20.0 & 0.381 \\
\hline Interpersonal violence & 3 & 10.0 & 3 & 10.0 & 1.000 \\
\hline Home accidents & 2 & 6.7 & 3 & 10.0 & 0.666 \\
\hline
\end{tabular}


Table (3): Distribution of patients according to signs and symptoms of mandibular fracture for the study and control groups $(\mathrm{n}=60)$.

\begin{tabular}{|c|c|c|c|c|c|c|c|c|c|}
\hline \multirow{3}{*}{$\begin{array}{l}\text { Signs and symptoms of } \\
\text { mandibular fracture }\end{array}$} & \multicolumn{4}{|c|}{$\operatorname{Study}(\mathrm{n}=\mathbf{3 0})$} & \multicolumn{4}{|c|}{ Control $(\mathrm{n}=30)$} & \multirow{3}{*}{ P. value } \\
\hline & \multicolumn{2}{|c|}{ Present } & \multicolumn{2}{|c|}{ Absent } & \multicolumn{2}{|c|}{ Present } & \multicolumn{2}{|c|}{ Absent } & \\
\hline & No. & $\%$ & No. & $\%$ & No. & $\%$ & No. & $\%$ & \\
\hline Pain & 30 & 100.0 & 0 & 0.0 & 30 & 100.0 & 0 & 0.0 & - \\
\hline Swelling & 29 & 96.7 & 1 & 3.3 & 30 & 100.0 & 0 & 0.0 & 0.313 \\
\hline Bleeding & 22 & 73.3 & 8 & 26.7 & 17 & 56.7 & 13 & 43.3 & 0.176 \\
\hline Facial bruising & 29 & 96.7 & 1 & 3.3 & 30 & 100.0 & 0 & 0.0 & 0.313 \\
\hline Limited jaw movement & 30 & 100.0 & 0 & 0.0 & 30 & 100.0 & 0 & 0.0 & - \\
\hline Malocclusion & 15 & 50.0 & 15 & 50.0 & 12 & 40.0 & 18 & 60.0 & 0.436 \\
\hline Step deformity & 10 & 33.3 & 20 & 66.7 & 9 & 30.0 & 21 & 70.0 & 0.781 \\
\hline Tenderness & 30 & 100.0 & 0 & 0.0 & 30 & 100.0 & 0 & 0.0 & - \\
\hline $\begin{array}{l}\text { Diminished or altered sensation to } \\
\text { lower lip (Numbness) }\end{array}$ & 13 & 43.3 & 17 & 56.7 & 9 & 30.0 & 21 & 70.0 & 0.284 \\
\hline Loss or damaged teeth & 17 & 56.7 & 13 & 43.3 & 22 & 73.3 & 8 & 26.7 & 0.176 \\
\hline
\end{tabular}

Table (4): Knowledge level for patients about nutrition, oral care and jaw exercises between study and control group pre - implementing nursing teaching protocol $(n=60)$.

\begin{tabular}{|c|c|c|c|c|c|c|c|c|c|}
\hline \multirow{3}{*}{ Variable } & \multicolumn{4}{|c|}{ Study $(\mathrm{n}=30)$} & \multicolumn{4}{|c|}{ Control $(n=30)$} & \multirow{3}{*}{ P. value } \\
\hline & \multicolumn{2}{|c|}{ Yes } & \multicolumn{2}{|c|}{ No } & \multicolumn{2}{|c|}{ Yes } & \multicolumn{2}{|c|}{ No } & \\
\hline & No. & $\%$ & No. & $\%$ & No. & $\%$ & No. & $\%$ & \\
\hline $\begin{array}{l}\text { Do you know how you eat } \\
\text { through intermaxillary fixation? }\end{array}$ & 11 & 36.7 & 19 & 63.3 & 5 & 16.7 & 25 & 83.3 & 0.080 \\
\hline $\begin{array}{l}\text { Do you know important nutrients } \\
\text { must eat to promote healing } \\
\text { process? }\end{array}$ & 9 & 30.0 & 21 & 70.0 & 10 & 33.3 & 20 & 66.7 & 0.781 \\
\hline $\begin{array}{l}\text { Do you know how you prepare } \\
\text { foods? }\end{array}$ & 5 & 16.7 & 25 & 83.3 & 1 & 3.3 & 29 & 96.7 & 0.085 \\
\hline $\begin{array}{l}\text { Do you know types of foods must } \\
\text { eat through Intermaxillary } \\
\text { fixation? }\end{array}$ & 8 & 26.7 & $2 r$ & 73.3 & 2 & 6.7 & 28 & 93.3 & $0.038^{*}$ \\
\hline $\begin{array}{l}\text { Do you experience weight loss } \\
\text { after postoperative period of } \\
\text { Intermaxillary fixation? }\end{array}$ & 1 & 3.3 & 29 & 96.7 & 0 & 0.0 & 30 & 100.0 & 0.313 \\
\hline Do you clean your teeth? & 20 & 66.7 & 10 & 33.3 & 14 & 46.7 & 16 & 53.3 & 0.118 \\
\hline $\begin{array}{l}\text { If yes; do you know the different } \\
\text { techniques for brushing teeth? }\end{array}$ & 7 & 23.3 & 23 & 76.7 & 3 & 10.0 & 27 & 90.0 & 0.166 \\
\hline $\begin{array}{l}\text { Are you aware that poor oral } \\
\text { care affect oral health? }\end{array}$ & 24 & 80.0 & 6 & 20.0 & 14 & 46.7 & 16 & 53.3 & $0.007 * *$ \\
\hline \multicolumn{10}{|l|}{ How do you clean your teeth? } \\
\hline -Toothbrush and toothpaste & 12 & 40.0 & - & - & 7 & 23.3 & - & - & \multirow{3}{*}{0.222} \\
\hline -Toothbrush and powder & 10 & 33.3 & - & - & 9 & 30.0 & - & - & \\
\hline$-\mathrm{No}$ & 8 & 26.7 & - & - & 14 & 46.7 & - & - & \\
\hline \multicolumn{10}{|l|}{$\begin{array}{l}\text { How many do you brush your } \\
\text { teeth each day? }\end{array}$} \\
\hline -Once & 2 & 9.1 & - & - & 7 & 43.8 & - & - & \multirow{4}{*}{0.338} \\
\hline -Twice & 12 & 54.5 & - & - & 3 & 18.8 & - & - & \\
\hline -After every meal & 2 & 9.1 & - & - & 1 & 6.3 & - & - & \\
\hline -Sometimes & 6 & 27.3 & - & - & 5 & 31.3 & - & - & \\
\hline Do you know jaw exercises? & 0 & 0.0 & 30 & 100.0 & 0 & 0.0 & 30 & 100.0 & - \\
\hline
\end{tabular}




\begin{tabular}{|c|c|c|c|c|c|c|c|c|c|}
\hline \multirow{3}{*}{ Variable } & \multicolumn{4}{|c|}{$\operatorname{Study}(n=30)$} & \multicolumn{4}{|c|}{ Control $(n=30)$} & \multirow{3}{*}{ P. value } \\
\hline & \multicolumn{2}{|c|}{ Yes } & \multicolumn{2}{|c|}{ No } & \multicolumn{2}{|c|}{ Yes } & \multicolumn{2}{|c|}{ No } & \\
\hline & No. & $\%$ & No. & $\%$ & No. & $\%$ & No. & $\%$ & \\
\hline $\begin{array}{l}\text { Do you know the importance of } \\
\text { these exercises? }\end{array}$ & 0 & 0.0 & 30 & 100.0 & 0 & 0.0 & 30 & 100.0 & - \\
\hline $\begin{array}{l}\text { Do you know types of jaw } \\
\text { exercises? }\end{array}$ & 0 & 0.0 & 30 & 100.0 & 0 & 0.0 & 30 & 100.0 & - \\
\hline $\begin{array}{l}\text { Do you know when you must do } \\
\text { these exercises(Time)? }\end{array}$ & 0 & 0.0 & 30 & 100.0 & 0 & 0.0 & 30 & 100.0 & - \\
\hline $\begin{array}{l}\text { Do you know techniques of these } \\
\text { exercises? }\end{array}$ & 0 & 0.0 & 30 & 100.0 & 0 & 0.0 & 30 & 100.0 & - \\
\hline
\end{tabular}

* Statistically significant difference $(\mathrm{p}<0.05)$

** Statistically significant difference $(\mathrm{p}<0.01)$

Table(5): Knowledge level for patients about nutrition, oral care and jaw exercises between study and control group post - implementing nursing teaching protocol $(n=60)$.

\begin{tabular}{|c|c|c|c|c|c|c|c|c|c|}
\hline \multirow{3}{*}{ Variable } & \multicolumn{4}{|c|}{$\operatorname{Study}(n=30)$} & \multicolumn{4}{|c|}{ Control $(\mathrm{n}=30)$} & \multirow{3}{*}{ P. value } \\
\hline & \multicolumn{2}{|c|}{ Yes } & \multicolumn{2}{|c|}{ No } & \multicolumn{2}{|c|}{ Yes } & \multicolumn{2}{|l|}{ No } & \\
\hline & No. & $\%$ & No. & $\%$ & No. & $\%$ & No. & $\%$ & \\
\hline $\begin{array}{l}\text { Do you know how you eat } \\
\text { through intermaxillary } \\
\text { fixation? }\end{array}$ & 26 & 86.7 & 4 & 13.3 & 6 & 20.0 & 24 & 80.0 & $0.001 * *$ \\
\hline $\begin{array}{l}\text { Do you know important } \\
\text { nutrients must eat to promote } \\
\text { healing process? }\end{array}$ & 24 & 80.0 & 6 & 20.0 & 12 & 40.0 & 18 & 60.0 & $0.002 * *$ \\
\hline $\begin{array}{l}\text { Do you know how you prepare } \\
\text { foods? }\end{array}$ & 22 & 73.3 & 8 & 26.7 & 1 & 3.3 & 29 & 96.7 & $0.001 * *$ \\
\hline $\begin{array}{l}\text { Do you know types of foods } \\
\text { must eat through } \\
\text { Intermaxillary fixation? }\end{array}$ & 27 & 90.0 & 3 & 10.0 & 7 & 23.3 & 23 & 46.7 & $0.001 * *$ \\
\hline $\begin{array}{l}\text { Do you experience weight loss } \\
\text { after postoperative period of } \\
\text { Intermaxillary fixation? }\end{array}$ & 19 & 63.3 & 11 & 36.7 & 3 & 10.0 & 27 & 90.0 & $0.001 * *$ \\
\hline Do you clean your teeth? & 29 & 96.7 & 1 & 3.3 & 13 & 43.3 & 17 & 56.7 & $0.001 * *$ \\
\hline $\begin{array}{l}\text { If yes; do you know the } \\
\text { different techniques for } \\
\text { brushing teeth? }\end{array}$ & 27 & 90.0 & 3 & 10.0 & 5 & 16.7 & 25 & 83.3 & $0.001 * *$ \\
\hline $\begin{array}{l}\text { Are you aware that poor oral } \\
\text { care affect oral health? }\end{array}$ & 30 & 100.0 & 0 & 0.0 & 14 & 46.7 & 16 & 53.3 & $0.001 * *$ \\
\hline \multicolumn{10}{|l|}{ How do you clean your teeth? } \\
\hline -Toothbrush and toothpaste & 27 & 90.0 & - & - & 7 & 23.3 & - & - & \multirow{3}{*}{$0.001 * *$} \\
\hline -Toothbrush and powder & 2 & 6.7 & - & - & 9 & 30.0 & - & - & \\
\hline -No & 1 & 3.3 & - & - & 14 & 46.7 & - & - & \\
\hline \multicolumn{10}{|l|}{$\begin{array}{l}\text { How many do you brush your } \\
\text { teeth each day? }\end{array}$} \\
\hline -Once & 0 & 0.0 & - & - & 7 & 43.8 & - & - & \multirow{4}{*}{$0.001 * *$} \\
\hline -Twice & 8 & 27.6 & - & - & 3 & 18.8 & - & - & \\
\hline -After every meal & 21 & 72.4 & - & - & 1 & 6.3 & - & - & \\
\hline -Sometimes & 0 & 0.0 & - & - & 5 & 31.3 & - & - & \\
\hline Do you know jaw exercises? & 28 & 93.3 & 2 & 6.7 & 2 & 6.7 & 28 & 93.3 & $0.001 * *$ \\
\hline $\begin{array}{l}\text { Do you know the importance of } \\
\text { these exercises? }\end{array}$ & 26 & 86.7 & 4 & 13.3 & 1 & 3.3 & 29 & 96.7 & $0.001 * *$ \\
\hline $\begin{array}{l}\text { Do you know types of jaw } \\
\text { exercises? }\end{array}$ & 30 & 100.0 & 0 & 0.0 & 0 & 0.0 & 30 & 100.0 & $0.001^{* *}$ \\
\hline $\begin{array}{l}\text { Do you know when you must } \\
\text { do these exercises(Time)? }\end{array}$ & 25 & 83.3 & 5 & 16.7 & 3 & 10.0 & 27 & 90.0 & $0.001 * *$ \\
\hline $\begin{array}{l}\text { Do you know techniques of } \\
\text { these exercises? }\end{array}$ & 29 & 96.7 & 1 & 3.3 & 2 & 6.7 & 28 & 93.3 & $0.001^{* *}$ \\
\hline
\end{tabular}


Table(6): Comparison between study and control groups knowledge level pre and post implementing nursing teaching protocol $(n=60)$.

\begin{tabular}{|c|c|c|c|c|c|c|c|c|c|c|}
\hline & \multicolumn{4}{|c|}{ pre } & \multirow{3}{*}{$\begin{array}{c}P . \\
\text { value }\end{array}$} & \multicolumn{4}{|c|}{ post } & \multirow{3}{*}{ P. value } \\
\hline & \multicolumn{2}{|c|}{$\begin{array}{c}\text { Study } \\
(\mathbf{n}=\mathbf{3 0})\end{array}$} & \multicolumn{2}{|c|}{$\begin{array}{c}\text { Control } \\
(\mathbf{n}=\mathbf{3 0})\end{array}$} & & \multicolumn{2}{|c|}{$\begin{array}{l}\text { Study } \\
(\mathbf{n}=\mathbf{3 0})\end{array}$} & \multicolumn{2}{|c|}{$\begin{array}{c}\text { Control } \\
(\mathbf{n}=\mathbf{3 0})\end{array}$} & \\
\hline & No. & $\%$ & No. & $\%$ & & No. & $\%$ & No. & $\%$ & \\
\hline \multicolumn{11}{|c|}{ Knowledge level } \\
\hline Satisfactory & 7 & 23.3 & 1 & 3.3 & \multirow[t]{2}{*}{$0.023^{*}$} & 27 & 90.0 & 4 & 13.3 & \multirow{2}{*}{$<0.001 * *$} \\
\hline Unsatisfactory & 23 & 76.7 & 29 & 96.7 & & 3 & 10.0 & 26 & 86.7 & \\
\hline
\end{tabular}

$*$ Statistically significant difference $(\mathrm{p}<0.05) \quad * *$ Statistically significant difference $(\mathrm{p}<0.01)$

Table (7): Comparison of late complications for both study and control group during hospitalization and on follow up after 2 and 4 weeks from discharge $(n=60)$.

\begin{tabular}{|c|c|c|c|c|c|}
\hline & Stu & 30) & Con & $1=30)$ & \\
\hline & No. & $\%$ & No. & $\%$ & P. value \\
\hline 1-Accomodation of feeding & & & & & \\
\hline During hospitalization & 29 & 96.7 & 30 & 100.0 & 0.313 \\
\hline After 2 weeks from discharge & 3 & 10.0 & 22 & 73.3 & $0.000 * *$ \\
\hline After 4 weeks & 0 & 0.0 & 5 & 16.7 & $0.020 *$ \\
\hline 2-Difficulty talking & & & & & \\
\hline During hospitalization & 24 & 80.0 & 25 & 83.3 & 0.739 \\
\hline After 2 weeks from discharge & 3 & 10.0 & 3 & 10.0 & 1.000 \\
\hline After 4 weeks & 0 & 0.0 & 1 & 3.3 & 0.313 \\
\hline 3-Periodontitis & & & & & \\
\hline During hospitalization & 2 & 6.7 & 1 & 3.3 & 0.991 \\
\hline After 2 weeks from discharge & 0 & 0.0 & 5 & 16.7 & 0.061 \\
\hline After 4 weeks & 0 & 0.0 & 4 & 13.3 & 0.121 \\
\hline 4-Gingivitis & & & & & \\
\hline During hospitalization & 7 & 23.3 & 3 & 10.0 & 0.166 \\
\hline After 2 weeks from discharge & 5 & 16.7 & 9 & 30.0 & 0.222 \\
\hline After 4 weeks & 0 & 0.0 & 5 & 16.7 & $0.020 *$ \\
\hline 5-Wound infection & & & & & \\
\hline During hospitalization & 8 & 26.7 & 16 & 53.3 & $0.035^{*}$ \\
\hline After 2 weeks from discharge & 6 & 20.0 & 18 & 60.0 & $0.002 * *$ \\
\hline After 4 weeks & 0 & 0.0 & 5 & 16.7 & $0.020^{*}$ \\
\hline $\begin{array}{l}\text { 6-Temporomandibular joint } \\
\text { movement }\end{array}$ & & & & & \\
\hline During hospitalization & 1 & 3.3 & 1 & 3.3 & 1.000 \\
\hline After 2 weeks from discharge & 15 & 50 & 17 & 56.7 & 0.794 \\
\hline After 4 weeks & 2 & 6.7 & 5 & 16.7 & 0.422 \\
\hline 7-Malocclusion & & & & & \\
\hline During hospitalization & 13 & 43.3 & 8 & 26.7 & 0.176 \\
\hline After 2 weeks from discharge & 10 & 33.3 & 11 & 36.7 & 0.787 \\
\hline After 4 weeks & 1 & 3.3 & 2 & 6.7 & 0.554 \\
\hline 8- Injury to inferior alveolar & & & & & \\
\hline During hospitalization & 11 & 36.7 & 8 & 26.7 & 0.405 \\
\hline After 2 weeks from discharge & 17 & 56.7 & 12 & 40.0 & 0.196 \\
\hline After 4 weeks & 17 & 56.7 & 11 & 36.7 & 0.121 \\
\hline 9-Injury to mental nerve & & & & & \\
\hline During hospitalization & 4 & 13.3 & 1 & 3.3 & 0.161 \\
\hline After 2 weeks from discharge & 9 & 30.0 & 4 & 13.3 & 0.117 \\
\hline After 4 weeks & 9 & 30.0 & 4 & 13.3 & 0.117 \\
\hline
\end{tabular}




\begin{tabular}{|l|c|c|c|c|c|}
\hline \multirow{2}{*}{} & \multicolumn{2}{|c|}{ Study $(\mathbf{n}=\mathbf{3 0})$} & \multicolumn{2}{c|}{ Control $(\mathbf{n}=\mathbf{3 0})$} & \multirow{2}{*}{ P. value } \\
\cline { 2 - 5 } & No. & $\mathbf{\%}$ & No. & \% & \\
\hline 10-Malunion & & & & & \\
\hline During hospitalization & 0 & 0.0 & 0 & 0.0 & - \\
After 2 weeks from discharge & 0 & 0.0 & 4 & 13.3 & $0.038^{*}$ \\
After 4 weeks & 0 & 0.0 & 0 & 0.0 & - \\
\hline 11-Nonunion & & & & & \\
\hline During hospitalization & 0 & 0.0 & 5 & 16.7 & 0.061 \\
After 2 weeks from discharge & 3 & 10.0 & 10 & 33.3 & 0.060 \\
After 4 weeks & 0 & 0.0 & 2 & 6.7 & 0.469 \\
\hline
\end{tabular}

Table (1): This table reveals that, as regard demographic characteristics; about half of the sample of the study and control group their age range between 18-29 years old, with mean of $(31.47 \pm 12.62$ and $32.6 \pm 13.55)$ respectively and about three-quarter of the study group were males $80.0 \%$, single $53.3 \%$, have secondary school $36.7 \%$ and farmer $23.3 \%$. While about three-quarter of the control group were males $76.7 \%$, married $53.3 \%$, illiterate $30.0 \%$ and drivers $30.0 \%$. As regard health habits; more than two third of the study group and control group used of tea/coffee $(86.7 \%$ and $73.3 \%)$ respectively.

Table (2): This table demonstrates that as regards, causes of mandibular fracture about half of the study and control group occurred as results of road traffic accident $(50.0 \%$ and $60.0 \%)$ respectively. There was no significant difference related to causes of mandibular fracture between both groups.

Table (3): This table shows that $100 \%$ of the patients in study and control groups had pain, limited jaw movement, and tenderness. The patients in control group more than study group as regard swelling, facial bruising, and loss or damaged teeth. There was no statistically significant difference between both groups as regard signs and symptoms of mandibular fracture.

Table (4): the highest percentage $(36.7 \%$ and $16.7 \%$ ) in study and control groups had knowledge about how eat through intermaxillary fixation. There is statistically significant difference between study and control groups as regard of patients aware that poor oral care affects oral health. They are equally in both groups and they haven't any knowledge about this item (jaw exercises).

Table (5): This table illustrates that there is statistically significant difference for the study group post implementing nursing teaching protocol as regards knowledge level of patients about nutrition, oral care, and jaw exercises.

Table (6): This table shows statistically significant difference between study and control group between study and control groups as regard knowledge level pre and posts implementing nursing teaching protocol.

Table (7): This table shows statistically significant difference between study and control group as regards late complications such as accommodation of feeding after 2 and 4 weeks from discharge (P. value $=0.000 * *, 0.020 *)$, gingivitis after 4 weeks from discharge $\left(\mathrm{P}\right.$. value $\left.=0.020^{*}\right)$, wound infection during hospitalization, after 2 and 4 weeks from discharge $\left(\mathrm{P}\right.$. value $\left.=0.035^{*}, 0.002^{* *}, 0.020^{*}\right)$, and malunion after 2 weeks from discharge $(\mathrm{P}$. value $=$ $0.038 *)$.

\section{Discussion:}

In recent years an increased incidence of maxillofacial trauma is observed (Cheema et al., 2006). Mandibular fractures are the commonest fractures encountered in maxillofacial trauma after nasal fractures. The most common causes of mandibular fractures are road traffic accidents, falls, missile injuries, assault and sports accidents. A clear understanding of the etiology and extent of all the maxillofacial injuries help in planning how to prevent or to reduce the number and severity of such injuries. The fractures of the mandible require early diagnosis, rapid and proper treatment and possible rehabilitation for optimum results (Gandhi et al., 2011).

The present study clarified that the incidence of mandibular fractures was higher in age group between 18 to 29 years. This finding consistent with (Hogg et al., 2000, Gassner et al., 2003, Eggensperger et al., 2007, Subhashraj et al., 2007, De Matos et al., 2010, Thorén et al., 2010, Ravindran and Ravindran Nair 2011) and differed with (Sakr et al., 2006) who revealed that higher incidence in first decade and also disagree with (Pappachan et al., 2006, Bakardjiev and Pechalova 2007, Lieger et al., 2009) who stated that the highest incidence of maxillofacial fractures is between the ages of 31 and 40. (King et al., 2004 and Bormann et al., 2009) mentioned that there was a high incidence of mandibular fractures in 
young populations. This can be explained by the fact that young adults have more social activity when compared with children or the elderly, which makes youth more susceptible to trauma and fractures (Chrcanovic et al., 2012). The mean age of the studied sample was $31.47 \pm 12.62,32.6 \pm 13.55$ for study and control groups respectively. This is in agreement with (Dongas and Hall 2002, Ahmed et al., 2004, Brasileiro and Passeri 2006).

In this study, the incidence of a male was more than female which is consistent with most of the studies (Subhashraj et al., 2007, Sakr et al., 2006 and Schön et al., 2001). This is most likely because men tend to participate in everyday activities that require more physical activity. Additionally, men more than women are involved in traffic accidents and violence.

As regard health habits more than two-thirds of the study group and control group used of tea/coffee and about half of the study and control group smokers. From a researcher point of views smoking considered as the main cause of delayed healing of jaw fracture. These results are in line with (Furto, 2006) who reported that the majority of sample were smokers and smoking is considered one of the risk factors for complications of surgical site infection. An adequate blood supply is also mandatory for fracture healing, so anything that diminishes blood flow (such as smoking or poor circulation) slows the healing process (Hamid et al., 2014).

In this study road traffic accidents (RTAs) was the leading cause of mandibular fractures and fall from height was the second cause, in agreement with (Sakr et al., 2006 and Adams et al., 2000) and disagree with (Schön et al., 2001and Lamphier et al., 2003) who reported that, the interpersonal violence was the main cause, as in the developed countries where the interpersonal violence appear to be the most common cause of mandibular fractures, where as in developing countries the road traffic accidents appear to be the most common cause (Subhashraj et al., 2007 and Sakr et al., 2006). In the developing countries, there is an ongoing migration from the rural to the busy urban environment where traffic volumes have increased rapidly and the infrastructure (e.g., road construction) is weak. Seat belts or helmets are not so widely accepted and deficiency in road traffic legislations, car maintenance, poor roads and bad driving - factors all contributing to a high incidence of road traffic accidents associated injuries (Ravindran and Ravindran Nair, 2011).

As regard signs and symptoms of mandibular fractures the current study showed that there was $(100 \%)$ of the sample had pain, limited jaw movement and tenderness and the patients in control group more than study group as regard swelling, in agreement with (Stacey et al.,2006 and Schneider et al.,2008) who suggested that less movement in the injured area results in less soft tissue edema. The finding indicated that there was no statistically significant difference between both groups as regard signs and symptoms of mandibular fractures.

The present study mentioned that, there is statistically significant difference between study and control group after implementing nursing teaching protocol as regard knowledge level of patients about nutrition, oral care, and jaw exercises. In my opinion, it's important to assess knowledge of patients because post treatment care included nutrition consultation of jaw-wire diet, oral hygiene, and physical therapy affect positively on patients outcomes and reduce incidence of complications as cleared in this study, the complications of study group decreased with following nursing teaching protocol.

The present study showed statistically significant difference between study and control groups as regard late complications, such as accommodation of feeding after 2 and 4 weeks from discharge, this results in agreement with (Fashola et al., 2003) who reported that inability to chew $(90.3 \%)$ was the commonest reason for seeking treatment, gingivitis after 4 weeks from discharge and wound infection during hospitalization, after 2 and 4 weeks from discharge in agreement with (Fox and Kellman, 2003) who mentioned that infection contribute significantly to the high prevalence of delayed and nonunion and the high incidence of infection may be due to long delay between injury and definitive treatment, the absence of prophylactic antibiotic therapy, unclean environment and lack of wound hygiene, chronic diseases such as diabetes and smoking was observed as the important patientrelated risk factor responsible for postoperative infection. In contrast with (Ellis, 1996) who noted that fibrous union is a common complication of infection associated with rigid internal fixation, which attributed to a lack of osseous tissue formation caused by an induced hypoxic environment. It was observed in this study that during the follow-ups, hygiene was maintained by majority of patients. Also, the present study reported statistically significant difference between study and control groups as regard malunion after 2 weeks from discharge in agreement with (Lumphier et al., 2003) who stated that early jaw movement and not following instructions increase incidence of malunion. 


\section{Conclusions}

Patients' knowledge regarding post-treatment care as (nutrition, oral hygiene, and jaw exercises) were inadequate. So a specialized nursing teaching protocol was designed, implemented and evaluated. Study group showed improvement in wound healing, maintain normal body weight and decreased incidence of complications while control group showed increase in complications such as gingivitis, periodontitis, wound infection, delayed wound healing and temporomandibular joint painful movement.

\section{Recommendations}

Based on the findings of the present study the following recommendations are suggested:

1. Awareness programs for patients who have had mandibular fractures and require additional verbal and written information about protecting their wound from infection.

2. Pamphlets and simple illustration booklets should be available for illiterate patients to provide them with simple explanation about safely living and maintaining normal body weight.

3. The patients must understand the importance of oral hygiene and visit a dentist regularly because dental infections can affect the wound healing and occurrence of complications.

4. Tell the patients about the importance of regular follow-up at regular times.

\section{References}

1. Adeyemo, W., Iwegbu, I., Bello, S., Okoturo, E., Olaitan, A., Ladeinde, A. (2008): Management of mandibular fractures in a developing country: a review of 314 cases from two urban centers in Nigeria. World journal of surgery. 32(12):pp.2631-2635.

2. Adams, J. (2012): Emergency Medicine: Clinical Essentials (Expert Consult-Online): $2^{\text {nd }}$ ed. , Elsevier Health Sciences, chapter 27, p.671.

3. Flint, P., Haughey, B., Robbins, K., Thomas, J., Niparko, J., Lund, V. (2014): Cummings otolaryngology-head and neck surgery: $6^{\text {th }}$ ed., Elsevier Health Sciences, chapter 2, p.1386.

4. Ambreen, A., and Shah, R. (2001): Causes of maxillofacial injuries - a three years study. J Surg Pak. 6(4):pp.25-27.

5. Bank, P. and Brown, A. (2001): Fractures of the facial skeleton. Wright. Oxford, Boston Banks PA (1998) Pragmatic approach to the management of condylar fractures Int $\mathbf{J}$ Oral Maxillofac Surg. 27:pp.244-246.

6. Subhashraj, K., Nandakumar, N. and Ravindran, C. (2007): Review of maxillofacial injuries in Chennai, India: a study of 2748 cases. British Journal of Oral and Maxillofacial Surgery. 45(8): pp.637-639.

7. Chrcanovic BR, Freire-Maia B, Souza LN, Araújo VO, Abreu MH. (2004): Facial fractures: a 1- year retrospective study in a hospital in Belo Horizonte. Braz Oral Res; 18(4): 322-328.

8. Cheema, S. and Amin, F. (2006): Incidence and causes of maxillofacial skeletal injuries at Mayo Hospital in Lahore, Pakistan. $\mathrm{Br} \mathrm{J}$ Oral Maxillofac Surg. 44:pp.232-234.

9. Gandhi, S., Ranganathan, L., Solanki, M., Mathew, G., Singh, I., and Bither, S. (2011): Pattern of maxillofacial fractures at a tertiary hospital in northern India: a 4-year retrospective study of 718 patients. Dent Traumatol. 27:pp.257-562.

10. Hogg, N., Stewart, T., Armstrong, J. and Girotti, M.(2000): Epidemiology of maxillofacial inuries at trauma hospitals in Ontario, Canada, between 1992 and 1997. J Trauma, 49: pp. 425-432.

11. Gassner, R., Tuli, T., Hächl, O., Rudisch, A. and Ulmer, H. (2003): Cranio-maxillofacial trauma: a 10-year review of 9543 cases with 21 067 injuries. J.Craniomaxillofac. Surg. 31: pp. 51-61.

12. Eggensperger, N., Smolka, K., Scheidegger, B., Zimmermann, H. and Iizuka, T. (2007): A 3-year survey of assault-related maxillofacial fractures in central Switzerland. $\mathbf{J}$ Craniomaxillofacial Surg. 35: pp.161-167.

13. De Matos, F., Arnez, M.., Sverzut, C., and Trivellato, A.E. (2010): A retrospective study of mandibular fracture in a 40-month period. Int J Oral Maxillofac Surg., 39: pp.10-15.

14. Thorén, H., Snäll, J., Salo, J., SuominenTaipale, L., Kormi, E., Lindqvist, C. and Törnwall, J. (2010): Occurrence and types of associated injuries in patients with fractures of the facial bones. J Oral Maxillofac Surg., 68:pp.805-810.

15. Ravindran, V. and Ravindran Nair, $K$. (2011): Meta-analysis of maxillofacial trauma in the northern districts of Kerala: One-year prospective study. J Maxillofac Oral Surg. 10: pp.321-327.

16.Sakr, K., Farag, I., and Zeitoun, I. (2006): Review of 509 mandibular fractures treated at the University Hospital, Alexandria, Egypt. 
British Journal of Oral and Maxillofacial Surgery. 44(2):pp.107-111.

17. Pappachan, B. and Alexander, M. (2006): Correlating facial fracture and cranial injuries. $\mathbf{J}$ Oral Maxillofac Surg. 64:pp.1023-1029.

18. Bakardjiev, A. and Pechalova, P.(2007): Maxillofacial fractures in Southern Bulgaria-A retrospective study of 1706 cases. J Craniomaxillofac Surg. 35:pp.147-150.

19. Lieger, O., Zix, J., Kruse, A. and Iizuka, T.(2009): Dental injuries in association with facial fractures. J Oral Maxillofac Surg. 67:pp.1680-1684.

20. King, R., Scianna, J., and Petruzzelli, G.J. (2004): Mandible fracture patterns: a suburban trauma center experience. Am J Otolaryngol. 25:pp.301-317.

21. Bormann, K., Wild, S., Gellrich, N., Kokemüller, H., Stühmer, C. (2009): a Fiveyear retrospective study of mandibular fractures in Freiburg, Germany: incidence, etiology, treatment, and complications. J Oral MaxillofacSurg. 67:pp.1251-1255.

22. Chrcanovic, B., Abreu, M., Freire-Maia, B.(2012): mandibular fractures: a 3 -year study in a hospital in Belo Horizonte, Brazil. J Craniomaxillofac Surg. 40:pp.116-123.

23. Dongas, P. and Hall, G.(2002): Mandibular fracture patterns in Tasmania, Australia, Australian Dental Journal, vol. 47, no. 2, pp.131137.

24. Ahmed, H. , Jaber, M., Abu Fanas, S. H., and Karas, M. (2004): The pattern of maxillofacial fractures in Sharjah, United Arab Emirates: a review of 230 cases, Oral Surgery, Oral Medicine, Oral Pathology, Oral Radiology and Endodontology, vol. 98, no.2, pp.166-170.

25. Brasileiro, B., and Passeri, L. (2006): "Epidemiological analysis of maxillofacial fractures in Brazil: a 5-year prospective study," Oral Surgery, Oral Medicine, Oral Pathology, Oral Radiology and Endodontology, vol. 102, no.1, pp.28-34.

26.Schön, R., Roveda, S. and Carter, B. (2001): Mandibular fractures in Townsville, Australia: incidence, aetiology, and treatment using the 2.0 AO/ASIF miniplate system. $\mathrm{Br} \mathrm{J}$ Oral MaxillofacSurg, 39:pp.145-148.

27. Furto, E., Cleland, J., Whitman, J. and Olson, K. (2006): Manual physical therapy interventions and exercise for patients with temporomandibular disorders. Cranio. 24:pp.283-291.

28. Hamid, H., Azilina, A., Igzeer, Y. (2014): The role of protein deficiency in the healing of mandibular fractures in a rabbit model.
International Journal of Pharmacy and Pharmaceutical Sciences. 6(2): pp.351-352.

29. Adams, C., Januszkiewcz, J. and Judson, J. (2000): Changing patterns of severe craniomaxillofacial trauma in Auckland over eight years. Aust N Z J. Surg. 70:pp.401-404.

30. Lamphier, J., Ziccardi, V., Ruvo, A. (2003): Complications of mandibular fractures in an urban teaching center. J Oral MaxillofacSurg. 61:Pp.745-749.

31. Stacey, D., Doyle, J., Mount, D., Snyder, M.C. (2006): Management of mandible fractures. Plast Reconstr Surg, 117(3):pp.48-60.

32. Schneider, M. , Erasmus, F. , Gerlach, K., Kuhlisch, E. (2008): Open renduction and internal fixation versus closed treatment and mandibulomaxillary fixation of fractures of the mandibular condylar process: a randomized, prospective, multicenter study with special evaluation of fracture level; J Oral Maxillofac Surg, 25:pp. 37-44.

33. Fashola, A., Obiechina, A., Arotiba, J. et al., (2003): Incidence and pattern of maxillofacial fractures in the elderly. Int J Oral Maxillofac Surg, 32:pp.206-208.

34. Fox, A. and Kellman, R. (2003): Mandibular Angle Fractures, Two Miniplate Fixation and Complications Arch Facial Plast., (6):pp.464469.

35. Ellis, E.(1996):Complications of rigid internal fixation for mandibular fractures. J Cranio Maxillofac Trauma, 2:pp.32-39. 